World Scientific Series in 20th Century Physics - Vol. 35




Published

Vol. 21 Spectroscopy with Coherent Radiation - Selected Papers of Norman F. Ramsey (with Commentary) edifed by N. F. Ramsey

Vol. 22 A Quest for Symmetry - Selected Works of Bunji Sakita edited by K. Kikkawa, M. Virasoro and S. R. Wadia

Vol. 23 Selected Papers of Kun Huang (with Commentary) edited by B. $F$. Zhu

Vol. 24 Subnuclear Physics - The First 50 Years: Highlights from Erice to ELN by A. Zichichi edited by O. Barnabei, P. Pupillo and F. Roversi Monaco

Vol. 25 The Creation of Quantum Chromodynamics and the Effective Energy by V. N. Gribov, G. 't Hooft, G. Veneziano and V. F. Weisskopf edited by L. N. Lipatov

Vol. 26 A Quantum Legacy - Seminal Papers of Julian Schwinger edited by $K$. A. Milton

Vol. 27 Selected Papers of Richard Feynman (with Commentary) edited by L. M. Brown

Vol. 28 The Legacy of Léon Van Hove edited by $A$. Giovannini

Vol. 29 Selected Works of Emil Wolf (with Commentary) edited by $E$. Wolf

Vol. 30 Selected Papers of J. Robert Schrieffer - In Celebration of His 70th Birthday edited by N. E. Bonesteel and L. P. Gor'kov

Vol. 31 From the Preshower to the New Technologies for Supercolliders - In Honour of Antonino Zichichi edited by B. H. Wiik, $A$. Wagner and $H$. Wenninger

Vol. 32 In Conclusion - A Collection of Summary Talks in High Energy Physics edited by J. D. Bjorken

Vol. 33 Formation and Evolution of Black Holes in the Galaxy - Selected Papers with Commentary edited by H. A. Bethe, G. E. Brown and C.-H. Lee

Vol. 35 A Career in Theoretical Physics, 2nd Edition by P. W. Anderson

For information on Vols. 1-20, please visit http://www.worldscibooks.com/series/wsscp_series.shtml 
World Scientific Series in 20th Century Physics - Vol. 35

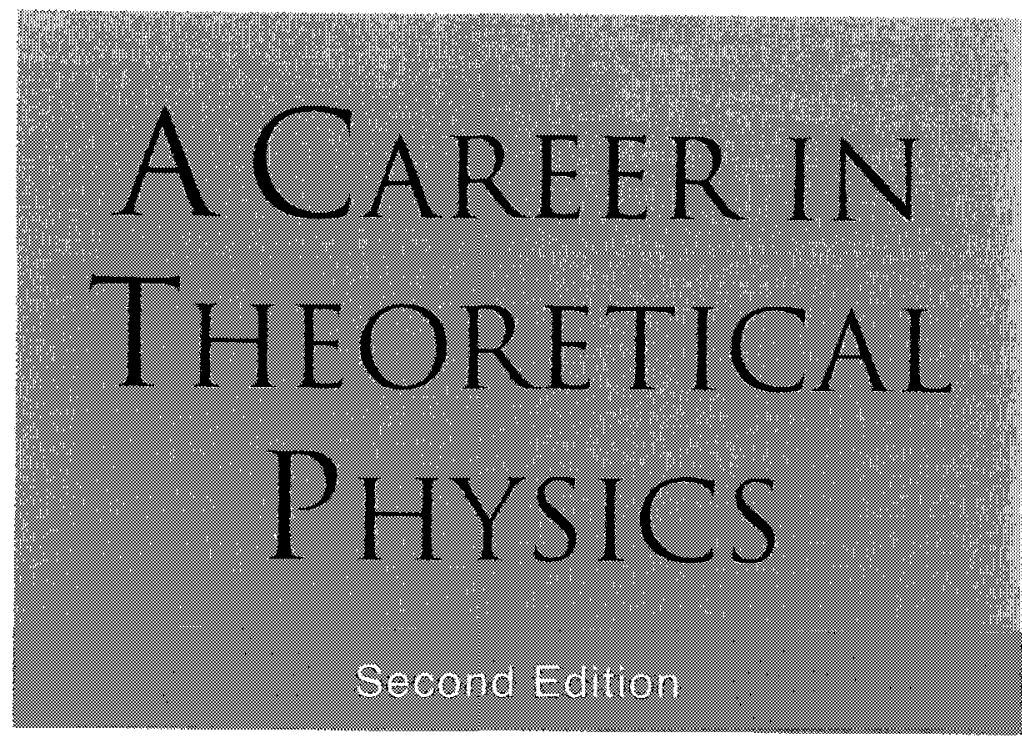

\section{PHILIP W. ANDERSON Princeton University, USA}




\section{Published by}

World Scientific Publishing Co. Pte. Ltd.

5 Toh Tuck Link, Singapore 596224

USA office: 27 Warren Street, Suite 401-402, Hackensack, NJ 07601

UK office: 57 Shelton Street, Covent Garden, London WC2H 9HE

The author and publisher would like to thank the following publishers for their permission to reproduce the reprinted papers found in this volume:

American Association for the Advancement of Science (Science)

American Institute of Physics (AIP Conf. Proc., Physics Today)

American Physical Society (Phys. Rev., Phys. Rev. Lett., Rev. Mod. Phys.)

Birkhauser Verlag (Ann. Henri Poincaré)

EDP Sciences (J. de Physique Colloque)

Elsevier Science Publishers (J. Phys. Chem. Solids, Solid State Commun., Physica, Phys. Reports, Stud. Hist. Phil. Mod. Phys.)

Institute of Physics (J. Phys. F, J. Phys. Condens. Matter)

Perseus Books (Emerging Syntheses in Science)

Springer-SBM (Valence Instabilities and Related Narrow Band Phenomena,

Proc. of 1983 NATO/CAP Inst. "Moment Formation in Solids", The Universality of Physics:

A Festschrift in honor of Deng Feng Wang)

Taylor and Francis [http://www.tandf.co.uk/journals] (Philosophical Magazine, Solid State Physics, Comments on Solid State Physics, Transition Metal Compounds)

To those publishers who have not granted us permission before publication, we have taken the liberty to reproduce their articles without consent. However we shall acknowledge them in future editions of this work.

\section{Library of Congress Cataloguing-in-Publication Data}

Anderson, P. W. (Philip W.), 1923-

A career in theoretical physics (2nd ed.) / Philip W. Anderson.

p. cm. -- (World Scientific series in 20th century physics ; v. 35)

ISBN 981-238-865-6 -- ISBN 981-238-866-4 (pbk)

1. Mathematical physics. I. Title. II. Series.

QC20.A55 2004

2004-063652

$530.1--\mathrm{dc} 22$

CIP

Copyright $\odot 2004$ this edition by World Scientific Publishing Co. Pte. Ltd.

All rights reserved.

Printed in Singapore. 


\section{PREFACE TO THE SECOND EDITION}

The plan which informed the selection of papers in the original volume was to stick as far as possible to summaries and reviews, since there was and is no possibility of covering all possible subjects. I think this may not have been a very good idea, since it slights the original research papers with their greater historical value. This volume is the only summary of my career likely to be published - after all, consider its title and therefore I have omitted some of these reviews, and substituted some of the research papers - for instance, the paper on line broadening which was a summary of my thesis, and the original paper on localization theory.

There is a second somewhat more embarrassing reason for this revision. At the time of the earlier edition, I was gung-ho for a theory of the mechanism of high $T_{\mathrm{c}}$ superconductivity which was shortly proven not to be the primary one. Among the "Central Dogmas" which I proudly reprinted in the book, number V was just plain wrong. I now belatedly understand what led me astray, but only because I was conclusively shown to be wrong, in the gentlest possible manner, by the careful experimental work of several colleagues, notably Kam Moler and collaborators. Fortunately, the correct theory is also partly based on the ideas I expressed in 1987, so not all is lost; but the papers in this section - involving nearly ten years of my professional life - have had to be mostly replaced, and some of the commentary rewritten. 
This page is intentionally left blank 


\section{INTRODUCTORY ESSAY TO THE FIRST EDITION}

It was in the winter of 1966-7 that I first tried to express my philosophy of what was important in science - or at least, of what was important that was possible for me. I has been invited to spend a month in the pleasant climate of La Jolla as the Regents' lecturer at the UCSD, visiting a department staffed by many of my old friends from Bell Labs, such as Bernd Matthias, Harry Suhl, and George Feher, who had been recruited by Walter Kohn, who was also by courtesy a Bell alumnus. One of the lectures I gave was called "More is Different". The original version, now lost, went over with the audience like a lead balloon, at least according to that portion of the audience most closely related to me. But a cleaned up version, which was published in Science in 1971, has been surprisingly influential. I have chosen to reprint it, out of chronological order, as an additional introduction to this collection.

I already had this overall vision, if only very vaguely, as I completed my thesis and other early work on the breadths of spectral lines. This work was among the first to approach what was normally thought of as a dissipative process and dealt with by Boltzmann equations for the microscopic variables, in terms of the macroscopic moments of the system as a whole. In my own mind called it the "one big molecule" method. I was beginning to see that somehow, starting from the fundamental atomiclevel laws of quantum theory, and without any more or less mystical appeals to the difference between the atomic domain and the macroscopic domain where deterministic Newtonian physics held way, one could really understand many of the properties of matter in that macroscopic domain. I also experienced two other insights: that these properties were often extraordinary; and that this understanding was often intellectually challenging and deep, at a level which was for me at least more exciting and worthwhile than any other purely intellectual exercise.

Thus I was launched into the search for the "Aufbauprinzips" of the everyday world. I admired Fröhlich's book on dielectric theory because it took the same point of view; I encountered it in my early work on ferroelectrics. Much more important, I came to see the crucial importance of the concept of broken symmetry, which has been a lifelong interest. Broken symmetry is the clearest instance of the process of emergence which lies behind "More is different". My interest began with the antiferromagnetic ground state work in 1952-3, from which I developed the concept of the Goldstone boson. In applying similar thinking to superconductivity, the Higgs 
phenomenon and the concept of Generalized Rigidity arose, the latter referring to the similarities between superfluidity, superconductivity, and elastic rigidity of all sorts. The topological theory of defects and dissipation is another facet of the conceptual structure of broken symmetry.

The first natural direction in which to seek for understanding of the real world is the explicit quantitative calculation of properties of real materials. This enterprise is particularly rewarding when the basic principles which govern these properties are new, or when the effects are subtle. This has been the mainspring of much of my work, from the early work on magnetic resonance and other spectra, to superexchange, the microscopic theory of superconductivity, and even work on the electronic structure of solids, foreshadowing the corrections to local density theory, and a paper where the idea is to develop a method which explains and exploits the strong locality of the chemical bond, perhaps the most fundamental observation in the field of chemistry, which nonetheless seems to interest quantum chemists not at all.

In 1956, stimulated by a pioneering paper on percolation by Hammersley and by some puzzling experiments, I started to look at the next level of complexity, those phenomena which are intrinsic to disorder. The first result was the theory of localisation of waves. This was slow of acceptance, and, perhaps discouraged by this fact, it was not until 1969 that I returned to it and also began to look at other disorder phenomena such as glass and spin glass. Spin glass stimulated a very fertile period of worldwide activity, first in the development of totally novel methodology, such as a new statistical mechanics of nonergodic systems, and then in applications of the new methodology to a "cornucopia" of new ideas and concepts and problems: neural networks, computer algorithms, rugged evolutionary landscapes, even immunology. This has left me involved in such fields as economics and the origin of life. The interest in complex, emergent phenomena made it natural for me to participate at an early stage in the Santa Fe Institute, founded on the twin principles of interdisciplinarity and of the importance and tractability of genuinely complex systems.

Left behind, in my old field of quantum condensed matter physics, was a worry about the validity and completeness of the theories of many-body quantum systems which had occupied that world in the 1950's and 60's. My work on the Anderson model of magnetic impurities had led on to the "infrared catastrophe" in Fermi systems which was the key to my work on the Kondo phenomenon which solved that isolated many-body system but only at the cost of going beyond the conventional perturbation theory. I was also disturbed by a class of strongly interacting superconducting materials, as well as our notable lack of success in dealing with the field of quantum valence fluctuations, i.e. "heavy electrons". With the appearance of 
the high $T_{\mathrm{c}}$ cuprates in 1987, my apprehensions became manifestly justified, and I began to formulate theories of metals based on new principles. This is the area which now most absorbs me, and where my efforts have been rewarded with the discovery of several new and fascinating phenomena. I reach my 70th year still involved in two major, very different types of problem. Although there is no easy way to round this one off, I have tried to give a flavor of the ideas involved.

My original vision seems to me to have been sound. The problem of understanding the "here and now" world seems to show no sign of being exhausted. The contribution of physics is the method of dealing correctly both with the substrate from which emergence takes place, and with the emergent phenomenon itself. Examples are legion: for one, superconductivity cannot be properly understood simply as a phenomenology without understanding electrons and their interactions, nor on the other hand as a property of a small number of electrons without taking into account the macroscopic system. Ever newer insights into the nature of the world around us will continually arise from this style of doing science. There will be physics even in a world without the SSC.

Regarding the choice of articles in this book: the sheer volume of either a complete selection of research articles or, even more, a complete set of reviews would have been much too great. I have given preference to materials which are for one reason or another not easy of access: lecture notes and out of print sources, for example. I have tried but failed to provide a selection of all major topics; the book is long enough. I have included a few items on history as I saw it, and on general aspects of science. 
This page is intentionally left blank 


\section{CONTENTS}

Preface to the Second Edition $\quad$ V

Introductory Essay to the First Edition vii

More Is Different 1

Science 177, 393-396 (1972)

1. Pressure Broadening in the Microwave and Infrared Regions 5 Phys. Rev. 76, 647-661 (1949)

2. Theory of Ferroelectric Behavior of Barium Titanate Ceramic Age 57, 29-34 (1951)

3. Use of Stochastic Methods in Line-Broadening Problems Kyoto Lectures, March 1954

4. An Approximate Quantum Theory of the Antiferromagnetic Ground State Phys. Rev. 86, 694-701 (1952)

5. Qualitative Considerations on the Statistics of the Phase Transition in $\mathrm{BaTiO}_{3}$-type Ferroelectrics

Conference Proceedings, Lebedev Physics Institute, USSR Academy of Sciences, Nov. 1958, Fizika Dielektrikov (Moscow), 1960, pp. 290-297

6. Ordering and Antiferromagnetism in Ferrites Phys. Rev. 102, 1008-1013 (1956)

7. Absence of Diffusion in Certain Random Lattices Phys. Rev. 109, 1492-1505 (1958)

8. New Method in the Theory of Superconductivity Phys. Rev. 110, 985-986 (1958) 
9. New Approach to the Theory of Superexchange Interactions Phys. Rev. 115, 2-13(1959)

10. Theory of Dirty Superconductors

J. Phys. Chem. Solids 11, 26-30 (1959)

11. Calculation of the Superconducting State Parameters with Retarded Electron-Phonon Interaction (with P. Morel)

Phys. Rev. 125, 1263-1271 (1962)

12. Generalized Bardeen-Cooper-Schrieffer States and the Proposed Low-Temperature Phase of Liquid $\mathrm{He}^{3}$ (with P. Morel) Phys. Rev. 123, 1911-1934 (1961)

13. Localized Magnetic States in Metals Phys. Rev. 124, 41-53 (1961)

14. Plasmons, Gauge Invariance, and Mass Phys. Rev. 130, 439-442 (1963)

15. Hard Superconductivity: Theory of the Motion of Abrikosov Flux Lines 175 (with Y.B. Kim)

Rev. Mod. Phys. 36, 39-43 (1964)

16. Probable Observation of the Josephson Superconducting

Tunneling Effect (with J. M. Rowell)

Phys. Rev. Lett. 10, 230-232 (1963)

17. Image of the Phonon Spectrum in the Tunneling Characteristic between Superconductors (with J. M. Rowell and D.E. Thomas)

Phys. Rev. Lett. 10, 334-336 (1963)

18. Exchange in Magnetic Insulators in Transition Metal Compounds, ed. E.R. Schatz, Buhl Int. Conference on Materials, Pittsburgh (Gordon and Breach, New York), 1964, pp. 17-28 
19. Superconductivity (Two Opinions)

(with B.T. Matthias)

Science, 144, 373-381 (1964)

20. Coherent Matter Field Phenomena in Superfluids in Some Recent Definitions in the Basic Sciences, Vol. 2, (Belfer Graduate School of Sciences, Yeshiva University, New York, 1969), ed. A. Gelbart, pp. 21-40

21. Considerations on the Flow of Superfluid Helium Rev. Mod. Phys. 38, 298-310 (1966)

22. Multiple-Scattering Theory and Resonances in Transition Metals (with W.L. McMillan) in Proc. of the Int. School of Physics 'Enrico Fermi' XXXVII (Academic Press, New York), 1967, pp. 50-86

23. Infrared Catastrophe in Fermi Gases with Local Scattering Potentials Phys. Rev. Lett. 18, 1049-1051 (1967)

24. The Kondo Effect I

Comments on Solid State Physics 1, no. 2, 31-36 (1968-69)

The Kondo Effect II

Comments on Solid State Physics 1, no. 6, 190-197 (1968-69)

Kondo Effect III: The Wilderness - Mainly Theoretical

Comments on Solid State Physics 3, no. 6, 153-158 (1971)

Kondo Effect IV: Out of the Wilderness

Comments on Solid State Physics 5, no. 3, 73-79 (1973)

25. Superconductivity in the Past and the Future in Superconductivity, Vol. 2, ed. R. Parks (Marcel Dekker, New York), 1968, pp. 1343-1358

26. Macroscopic Coherence and Superfluidity in Contemporary Physics, Vol. 1 (IAEA, Vienna), 1969, pp. 47-54 
27. The Fermi Glass: Theory and Experiment

Comments on Solid State Physics 2, 193-198 (1970)

28. Space-Time and Scaling Techniques in the Kondo Problem

in Proc. of $12^{\text {th }}$ Int. Conf. on Low Temperature Physics, ed. E. Kanda (Academic Press, 1971), pp. 657-660

29. Anomalous Low-temperature Thermal Properties of Glasses and Spin Glasses

(with B.I. Halperin and C.M. Varma)

Philos. Mag. 25, 1-9 (1972)

30. Comments on the Maximum Superconducting Transition Temperature

(with M.L. Cohen)

AIP. Conf. Proc., 1971, pp. 17-27

Comment on "Model for an Exciton Mechanism of Superconductivity"

(with J.C. Inkson)

Phys. Rev. B. 8, 4429-4432 (1973)

31. Resonating Valence Bonds: A New Kind of Insulator?

Materials Research Bulletin 8, 153-160 (1973)

32. Anisotropic Superfluidity in ${ }^{3} \mathrm{He}$ : A Possible Interpretation of

Its Stability as a Spin-Fluctuation Effect

(with W.F. Brinkman)

Phys. Rev. Lett. 30, 1108-1111 (1973)

33. Conference Summary

in Collective Properties of Physical Systems, eds. B. Lundqvist and

S. Lundqvist, Proc. of Nobel Symposium, Goteborg, Sweden,

13 June 1973 (Academic Press, 1974), pp. 266-271

34. Asymptotically Exact Methods in the Kondo Problem (with G. Yuval)

in Magnetism, vol. V, ed. H. Suhl (Academic Press, 1973), pp. 217-236 
35. Many-Body Effects at Surfaces

in Elementary Excitations in Solids, Molecules and Atoms

(Plenum, 1974), pp. 1-29

36. Conductivity from Charge or Spin Density Waves

(with P.A. Lee and T.M. Rice)

Solid State Communications 14, 703-709 (1974)

37. Uses of Solid State Analogies in Elementary Particle Theory in Proc. of Conf. on Gauge Theories and Modern Field Theory, eds. R. Arnowitt and P. Nath (MIT Press, 1976), pp. 311-335

38. Possible Consequences of Negative U Centers in Amorphous Materials J. de Physique Colloque, No. 4, 339--342 (1976)

39. Theory of Spin Glasses 509 (with S.F. Edwards)

J. Phys. F. 5, 965-974 (1975)

40. Solution of "Solvable Model of a Spin Glass" (with D.J. Thouless and R.G. Palmer)

Philos. Mag. 35, 593-601 (1977)

41. Phase Slippage without Vortex Cores: Vortex Textures in Superfluid ${ }^{3} \mathrm{He}$ 531 (with G. Toulouse)

Phys. Rev. Lett. 18, 508-511 (1977)

42. Scaling Theory of Localization: Absence of Quantum Diffusion in Two Dimensions

(with E. Abrahams, D.C. Licciardello and T.V. Ramakrishnan)

Phys. Rev. Lett. 42, 673-676 (1979)

43. Some General Thoughts about Broken Symmetry in Symmetries and Broken Symmetries in Condensed Matter Physics, ed. N. Boccara (IDSET, Paris, 1981), pp. 11-20 
44. The Rheology of Neutron Stars: Vortex Line Pinning in the Crust Superfluid

(with M.A. Alpar, D. Pines and J. Shaham)

Philos. Mag. A45, 227-238 (1982)

45. Localization Redux

Physica 117B \& 118B, 30-35 (1983)

46. New Method for Scaling Theory of Localization. II: Multi-Channel Theory of a "Wire" and Possible Extension to Higher Dimensionality Phys. Rev. B. 23, 4828-4836 (1981)

47. Definition and Measurement of the Electrical and Thermal Resistances (with H.-L. Engquist)

Phys. Rev. B. 24, 1151-1154 (1981)

48. Suggested Model for Prebiotic Evolution: The Use of Chaos

Proc. Natl. Acad. Sci. (USA) 80, 3386-3390 (1983)

49. Chemical Pseudopotentials

Phys. Reports 110, Nos. 5\&6, 311-319 (1984)

50. Spin Glass Hamiltonians: A Bridge between Biology, Statistical Mechanics and Computer Science in Emerging Syntheses in Science, ed. D. Pines (Addison-Wesley, 1987), pp. 17-20

51. Measurement in Quantum Theory and the Problem of Complex Systems

in The Lessons of Quantum Theory, ed. J. de Boer, E. Dal and

O. Ulfbeck, talk given at the Niels Bohr Centenary Symposium, Copenhagen, October 1985 (Elsevier Science, 1986), pp. 23-34

52. It's Not Over Till the Fat Lady Sings

Talk given at History of Superconductivity, APS Meeting, March 1987

53. Spin Glass I: A Scaling Law Rescued

Physics Today 41\#1, 9 (1988) 
Spin Glass II: Is There a Phase Transition?

Physics Today 41\#3, 9 (1988)

Spin Glass III: Theory Raises Its Head

Physics Today 41\#6, 9 (1988)

Spin Glass IV: Glimmerings of Trouble

Physics Today 41\#9, 9 (1988)

Spin Glass V: Real Power Brought to Bear

Physics Today 42\#7, 9 (1989)

Spin Glass VI: Spin Glass as Cornucopia

Physics Today 42\#9, 9 (1989)

Spin Glass VII: Spin Glass as Paradigm

Physics Today 43\#3, 9 (1990)

54. Epilogue

in Valence Instabilities and Related Narrow-band Phenomena,

ed. R.D. Parks (Plenum, 1977), pp. 389-396

55. Present Status of Theory: $1 / N$ Approach in Proc. of 1983 NATO/CAP Inst. "Moment Formation in Solids", ed. W.J.L. Buyers (Plenum), pp. 313-326

56. The Problem of Fluctuating Valence in f-Electron Metals in Windsurfing in the Fermi Sea, Vol. 1, eds. T.T.S. Kuo and J. Speth (Elsevier Science, 1987), pp. 61-67

57. Gutzwiller-Hubbard Lattice-Gas Model with Variable Density: Application to Normal Liquid ${ }^{3} \mathrm{He}$ (with D. Vollhardt and P. Wölfle)

Phys. Rev. B. 35, 6703-6715 (1987)

58. Some Ideas on the Aesthetics of Science

Lecture given at the $50^{\text {th }}$ Anniversary Seminar of the Faculty of Science and Technology, Keio University, Japan, May 1989 
59. Theoretical Paradigms for the Sciences of Complexity

Nishima Memorial Lecture, Department of Physics, Keio University, Japan, May 1989

60. 50 Years of the Mott Phenomenon: Insulators, Magnets, Solids, and Superconductors as Aspects of Strong-Repulsion Theory 723 in Frontiers and Borderlines in Many-Particle Physics, Proceedings of the Enrico International School of Physics, Varenna, July 1987 (North-Holland, 1987), pp. 1-40

61. Theories of Fullerene $T_{c}$ 's Which Will Not Work

62. The Reverend Thomas Bayes, Needles in Haystacks, and the Fifth Force 775 Physics Today 45\#1, 9 (1992)

63. The Eightfold Way to the Theory of Complexity: A Prologue in Complexity, eds. G. Cowan, D. Pines and D. Meltzer (Addison-Wesley, 1994), pp. 7-16

64. Magnetic Field Induced Confinement in Strongly Correlated Anisotropic Materials (with S.P. Strong and D.G. Clarke) Phys. Rev. Lett. 73, 1007-1010 (1994)

65. Physics: The Opening to Complexity 795 Proc. Natl. Acad. Sci. (USA) 92, 6653-6654 (1995)

66. Beyond Chaos: Singular Distributions and Power Laws

67. Essay Review - Science: A 'Dappled World' or a 'Seamless Web'? 809 Stud. Hist. Phil. Mod. Phys. 32, 487-494 (2001)

68. RVB Redux: A Synergistic Theory of High $\mathrm{T}_{\mathrm{c}}$ Cuprates in The Universality of Physics, A Festschrift in Honor of Deng Feng Wang, ed. Khuri et al. (Kluwer/Plenum, 2001), pp. 3-8 
69. Physics of the Pseudogap Phase of High $T_{c}$ Cuprates, or, RVB meets Umklapp

J. Phys. Chem. Solids 63, 2145-2148 (2002)

70. In Praise of Unstable Fixed Points: The Way Things Actually Work Physica B 318, 28-32 (2002)

71. Rise of Complexity, 1953-2002 Ann. Henri Poincaré 4, S1-S6 (2003)

72. The Physics behind High-temperature Superconducting Cuprates: The 'Plain Vanilla' Version of RVB (with P.A. Lee, M. Randeria, T. M. Rice, N. Trivedi and F.C. Zhang) J. Phys. Condens. Matter 16, R755-R769 (2004) 


\title{
More Is Different
}

\author{
Broken symmetry and the nature of \\ the hierarchical structure of science.
}

\author{
P. W. Anderson
}

The reductionist hypothesis may still be a topic for controversy among philosophers, but among the great majority of active scientists I think it is accepted without question. The workings of our minds and bodies, and of all the animate or inanimate matter of which we have any detailed knowledge, are assumed to be controlled by the same set of fundamental laws, which except under certain extreme conditions we feel we know pretty well.

It seems inevitable to go on uncritically to what appears at first sight to be an obvious corollary of reductionism: that if everything obeys the same fundamental laws, then the only scientists who are studying anything really fundamental are those who are working on those laws. In practice, that amounts to some astrophysicists, some elementary particle physicists, some logicians and other mathematicians, and few others. This point of view, which it is the main purpose of this article to oppose, is expressed in a rather wellknown passage by Weisskopf (1):

Looking at the development of science in the Twentieth Century one can distinguish two trends, which I will call "intensive" and "extensive" research, lacking a better terminology. In short: intensive research goes for the fundamental laws, extensive research goes for the ex-

The author is a member of the technical staft of the Bell Telephone Laboratories, Murray Hill New Jersey 07974, and visiting professor of theoretical physics at Cavendish Laboratory Cambridge, England. This article is an expanded version of a Regents' Lecture given in 1967 at the University of California, La Jolla. planation of phenomena in terms of known fundamental laws. As always, distinctions of this kind are not unambiguous, but they are clear in most cases. Solid state physics, plasma physics, and perhaps also biology are extensive. High energy physics and a good part of nuclear physics are intensive. There is always much less intensive research going on than extensive. Once new fundamental laws are discovered, a large and ever increasing activity begins in order to apply the discoveries to hitherto unexplained phenomena. Thus, there are two dimensions to basic research. The frontier of science extends all along a long line from the newest and most modern intensive research, over the extensive research recently spawned by the intensive research of yesterday, to the broad and well developed web of extensive research activities based on intensive research of past decades.

The effectiveness of this message may be indicated by the fact that I heard it quoted recently by a leader in the field of materials science, who urged the participants at a meeting dedicated to "fundamental problems in condensed matter physics" to accept that there were few or no such problems and that nothing was left but extensive science, which he seemed to equate with device engineering.

The main fallacy in this kind of thinking is that the reductionist hypothesis does not by any means imply a "constructionist" one: The ability to reduce everything to simple fundamental laws does not imply the ability to start from those laws and reconstruct the universe. In fact, the more the elementary particle physicists tell us about the nature of the fundamental laws, the less relevance they seem to have to the very real problems of the rest of science, much less to those of society.

The constructionist hypothesis breaks down when confronted with the twin difficulties of scale and complexity. The behavior of large and complex aggregates of elementary particles, it turns out, is not to be understood in terms of a simple extrapolation of the properties of a few particles. Instead, at each level of complexity entirely new properties appear, and the understanding of the new behaviors requires research which I think is as fundamental in its nature as any other. That is, it seems to me that one may array the sciences roughly linearly in a hierarchy, according to the idea: The elementary entities of science $X$ obey the laws of science $\mathrm{Y}$.
$\mathrm{X}$

solid state or many-body physics chemistry molecular biology cell biology

-

-

psychology social sciences
But this hierarchy does not imply that science $X$ is "just applied Y." At each stage entirely new laws, concepts, and generalizations are necessary, requiring inspiration and creativity to just as great a degree as in the previous one. Psychology is not applied biology, nor is biology applied chemistry.

In my own field of many-body physics, we are, perhaps, closer to our fundamental, intensive underpinnings than in any other science in which nontrivial complexities occur, and as a result we have begun to formulate a general theory of just how this shift from quantitative to qualitative differentiation takes place. This formulation, called the theory of "broken symmetry," may be of help in making more generally clear the breakdown of the constructionist converse of reductionism. I will give an elementary and incomplete explanation of these ideas, and then go on to some more general speculative comments about analogies at 
other levels and about similar phenomena.

Before beginning this I wish to sort out two possible sources of misunderstanding. First, when I speak of scale change causing fundamental change I do not mean the rather well-understood idea that phenomena at a new scale may obey actually different fundamental laws-as, for example, general relativity is required on the cosmological scale and quantum mechanics on the atomic. I think it will be accepted that all ordinary matter obeys simple electrodynamics and quantum theory, and that really covers most of what I shall discuss. (As I said, we must all start with reductionism, which I fully accept.) A second source of confusion may be the fact that the concept of broken symmetry has been borrowed by the elementary particle physicists, but their use of the term is strictly an analogy, whether a deep or a specious one remaining to be understood.

Let me then start my discussion with an example on the simplest possible level, a natural one for me because I worked with it when I was a graduate student: the ammonia molecule. At that time everyone knew about ammonia and used it to calibrate his theory or his apparatus, and I was no exception. The chemists will tell you that ammonia "is" a triangular pyramid

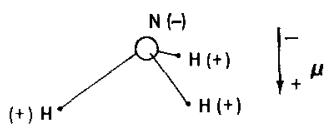

with the nitrogen negatively charged and the hydrogens positively charged, so that it has an electric dipole moment $(\mu)$, negative toward the apex of the pyramid. Now this seemed very strange to me, because I was just being taught that nothing has an electric dipole moment. The professor was really proving that no nucleus has a dipole moment, because he was teaching nuclear physics, but as his arguments were based on the symmetry of space and time they should have been correct in general.

I soon learned that, in fact, they were correct (or perhaps it would be more accurate to say not incorrect) because he had been careful to say that no stationary state of a system (that is, one which does not change in time) has an electric dipole moment. If ammonia starts out from the above unsymmetrical state, it will not stay in it very long. By means of quantum mechanical tunneling, the nitrogen can leak through the triangle of hydrogens to the other side, turning the pyramid inside out, and, in fact, it can do so very rapidly. This is the so-called "inversion," which occurs at a frequency of about $3 \times 10^{10}$ per second. A truly stationary state can only be an equal superposition of the unsymmetrical pyramid and its inverse. That mixture does not have a dipole moment. ( $I$ warn the reader again that $I$ am greatly oversimplifying and refer him to the textbooks for details.)

I will not go through the proof, but the result is that the state of the system, if it is to be stationary, must always have the same symmetry as the laws of motion which govern it. A reason may be put very simply: In quantum mechanics there is always a way, unless symmetry forbids, to get from one state to another. Thus, if we start from any one unsymmetrical state, the system will make transitions to others, so only by adding up all the possible unsymmetrical states in a symmetrical way' can we get a stationary state. The symmetry involved in the case of ammonia is parity, the equivalence of left- and right-handed ways of looking at things. (The elementary particle experimentalists' discovery of certain violations of parity is not relevant to this question; those effects are too weak to affect ordinary matter.)

Having seen how the ammonia molecule satisfies our theorem that there is no dipole moment, we may look into other cases and, in particular, study progressively bigger systems to see whether the state and the symmetry are always related. There are other similar pyramidal molecules, made of heavier atoms. Hydrogen phosphide, $\mathrm{PH}_{3}$, which is twice as heavy as ammonia, inverts, but at one-tenth the ammonia frequency. Phosphorus trifluoride, $\mathrm{PF}_{3}$, in which the much heavier fluorine is substituted for hydrogen, is not observed to invert at a measurable rate, although theoretically one can be sure that a state prepared in one orientation would invert in a reasonable time.

We may then go on to more complicated molecules, such as sugar, with about 40 atoms. For these it no longer makes any sense to expect the molecule to invert itself. Every sugar molecule made by a living organism is spiral in the same sense, and they never invert, either by quantum mechanical tunneling or even under thermal agitation at normal temperatures. At this point we must forget about the possibility of inversion and ignore the parity symmetry: the symmetry laws have been, not repealed, but broken.

If, on the other hand, we synthesize our sugar molecules by a chemical reaction more or less in thermal equilibrium, we will find that there are not, on the average, more left- than righthanded ones or vice versa. In the absence of anything more complicated than a collection of free molecules, the symmetry laws are never broken, on the average. We needed living matter to produce an actual unsymmetry in the populations.

In really large, but still inanimate, aggregates of atoms, quite a different kind of broken symmetry can occur, again leading to a net dipole moment or to a net optical rotating power, or both. Many crystals have a net dipole moment in each elementary unit cell (pyroelectricity), and in some this moment can be reversed by an electric field (ferroelectricity). This asymmetry is a spontaneous effect of the crystal's seeking its lowest energy state. Of course, the state with the opposite moment also exists and has, by symmetry, just the same energy, but the system is so large that no thermal or quantum mechanical force can cause a conversion of one to the other in a finite time compared to, say, the age of the universe.

There are at least three inferences to be drawn from this. One is that symmetry is of great importance in physics. By symmetry we mean the existence of different viewpoints from which the system appears the same. It is only slightly overstating the case to say that physics is the study of symmetry. The first demonstration of the power of this idea may have been by Newton, who may have asked himself the question: What if the matter here in my hand obeys the same laws as that up in the skythat is, what if space and matter are homogeneous and isotropic?

The second inference is that the internal structure of a piece of matter need not be symmetrical even if the total state of it is. I would challenge you to start from the fundamental laws of quantum mechanics and predict the ammonia inversion and its easily observable properties without going through the stage of using the unsymmetrical pyramidal structure, even though no "state" ever has that structure. It is fascinating that it was not until a couple of decades ago (2) that nuclear physicists stopped thinking of the nucleus as a featureless, symmetrical little ball and realized that while it really never has a dipole moment, it can become football- 
shaped or plate-shaped. This has observable consequences in the reactions and excitation spectra that are studied in nuclear physics, even though it is much more difficult to demonstrate directly than the ammonia inversion. In my opinion, whether or not one calls this intensive research, it is as fundamental in nature as many things one might so label. But it needed no new knowledge of fundamental laws and would have been extremely difficult to derive synthetically from those laws; it was simply an inspiration, based, to be sure, on everyday intuition, which suddenly fitted everything together.

The basic reason why this result would have been difficult to derive is an important one for our further thinking. If the nucleus is sufficiently small there is no real way to define its shape rigorously: Three or four or ten particles whirling about each other do not define a rotating "plate" or "football." It is only as the nucleus is considered to be a many-body system-in what is often called the $N \rightarrow \infty$ limit-that such behavior is rigorously definable. We say to ourselves: A macroscopic body of that shape would have such-and-such a spectrum of rotational and vibrational excitations, completely different in nature from those which would characterize a featureless system. When we see such a spectrum, even not so separated, and somewhat imperfect, we recognize that the nucleus is, after all, not macroscopic; it is merely approaching macroscopic behavior. Starting with the fundamental laws and a computer, we would have to do two impossible things - solve a problem with infinitely many bodies, and then apply the result to a finite system-before we synthesized this behavior.

A third insight is that the state of a really big system does not at all have to have the symmetry of the laws which govern it; in fact, it usually has less symmetry. The outstanding example of this is the crystal: Built from a substrate of atoms and space according to laws which express the perfect homogeneity of space, the crystal suddenly and unpredictably displays an entirely new and very beautiful symmetry. The general rule, however, even in the case of the crystal, is that the large system is less symmetrical than the underlying structure would suggest: Symmetrical as it is, a crystal is less symmetrical than perfect homogeneity.

Perhaps in the case of crystals this appears to be merely an exercise in confusion. The regularity of crystals could be deduced semiempirically in the mid-19th century without any complicated reasoning at all. But sometimes, as in the case of superconductivity, the new symmetry-now called broken symmetry because the original symmetry is no longer evident-may be of an entirely unexpected kind and extremely difficult to visualize. In the case of superconductivity, 30 years elapsed between the time when physicists were in possession of every fundamental law necessary for explaining it and the time when it was actually done.

The phenomenon of superconductivity is the most spectacular example of the broken symmetries which ordinary macroscopic bodies undergo, but it is of course not the only one. Antiferromagnets, ferroelectrics, liquid crystals, and matter in many other states obey a certain rather general scheme of rules and ideas, which some many-body theorists refer to under the general heading of broken symmetry. I shall not further discuss the history, but give a bibliography at the end of this article (3).

The essential idea is that in the socalled $N \rightarrow \infty$ limit of large systems (on our own, macroscopic scale) it is not only convenient but essential to realize that matter will undergo mathematically sharp, singular "phase transitions" to states in which the microscopic symmetries, and even the microscopic equations of motion, are in a sense violated. The symmetry leaves behind as its expression only certain characteristic behaviors, for instance, long-wavelength vibrations, of which the familiar example is sound waves; or the unusual macroscopic conduction phenomena of the superconductor; or, in a very deep analogy, the very rigidity of crystal lattices, and thus of most solid matter. There is, of course, no question of the system's really violating, as opposed to breaking, the symmetry of space and time, but because its parts find it energetically more favorable to maintain certain fixed relationships with each other, the symmetry allows only the body as a whole to respond to external forces.

This leads to a "rigidity," which is also an apt description of superconductivity and superfluidity in spite of their apparent "fluid" behavior. [In the former case, London noted this aspect very early (4).] Actually, for a hypothetical gaseous but intelligent citizen of Jupiter or of a hydrogen cloud somewhere in the galactic center, the properties of ordinary crystals might well be a more baffling and intriguing puzzle than those of superfluid helium.
I do not mean to give the impression that all is settled. For instance, I think there are still fascinating questions of principle about glasses and other amorphous phases, which may reveal even more complex types of behavior. Nevertheless, the role of this type of broken symmetry in the properties of inert but macroscopic material bodies is now understood, at least in principle. In this case we can see how the whole becomes not only more than but very different from the sum of its parts.

The next order of business logically is to ask whether an even more complete destruction of the fundamental symmetries of space and time is possible and whether new phenomena then arise, intrinsically different from the "simple" phase transition representing a condensation into a less symmetric state.

We have already excluded the apparently unsymmetric cases of liquids, gases, and glasses. (In any real sense they are more symmetric.) It seems to me that the next stage is to consider the system which is regular but contains information. That is, it is regular in space in some sense so that it can be "read out," but it contains elements which can be varied from one "cell" to the next. An obvious example is DNA; in everyday life, a line of type or a movie film have the same structure. This type of "information-bearing crystallinity" seems to be essential to life. Whether the development of life requires any further breaking of symmetry is by no means clear.

Keeping on with the attempt to characterize types of broken symmetry which occur in living things, I find that at least one further phenomenon seems to be identifiable and either universal or remarkably common, namely, ordering (regularity or periodicity) in the time dimension. A number of theories of life processes have appeared in which regular pulsing in time plays an important role: theories of development, of growth and growth limitation, and of the memory. Temporal regularity is very commonly observed in living objects. It plays at least two kinds of roles. First, most methods of extracting energy from the environment in order to set up a continuing, quasi-stable process involve time-periodic machines, such as oscillators and generators, and the processes of life work in the same way. Second, temporal regularity is a means of handling information, similar to information-bearing spatial regularity. Human spoken language is an example, and it 
is noteworthy that all computing machines use temporal pulsing. A possible third role is suggested in some of the theories mentioned above: the use of phase relationships of temporal pulses to handle information and control the growth and development of cells and organisms (5).

In some sense, structure-functional structure in a teleological sense, as opposed to mere crystalline shape-must also be considered a stage, possibly intermediate between crystallinity and information strings, in the hierarchy of broken symmetries.

To pile speculation on speculation, I would say that the next stage could be hierarchy or specialization of function, or both. At some point we have to stop talking about decreasing symmetry and start calling it increasing complication. Thus, with increasing complication at each stage, we go on up the hierarchy of the sciences. We expect to encounter fascinating and, I believe, very fundamental questions at each stage in fitting together less complicated pieces into the more complicated system and understanding the basically new types of behavior which can result.

There may well be no useful parallel to be drawn between the way in which complexity appears in the simplest cases of many-body theory and chemistry and the way it appears in the truly complex cultural and biological ones, except perhaps to say that, in general, the relationship between the system and its parts is intellectually a one-way street. Synthesis is expected to be all but im- possible; analysis, on the other hand, may be not only possible but fruitful in all kinds of ways: Without an understanding of the broken symmetry in superconductivity, for instance, Josephson would probably not have discovered his effect. [Another name for the Josephson effect is "macroscopic quantum-interference phenomena": interference effects observed between macroscopic wave functions of electrons in superconductors, or of helium atoms in superfluid liquid helium. These phenomena have already enormously extended the accuracy of electromagnetic measurements, and can be expected to play a great role in future computers, among other possibilities, so that in the long run they may lead to some of the major technological achievements of this decade (6).l For another example, biology has certainly taken on a whole new aspect from the reduction of genetics to biochemistry and biophysics, which will have untold consequences. So it is not true, as a recent article would have it (7), that we each should "cultivate our own valley, and not attempt to build roads over the mountain ranges .. between the sciences." Rather, we should recognize that such roads, while often the quickest shortcut to another part of our own science, are not visible from the viewpoint of one science alone.

The arrogance of the particle physicist and his intensive research may be behind us (the discoverer of the positron said "the rest is chemistry"), but we have yet to recover from that of some molecular biologists, who seem deter- mined to try to reduce everything about the human organism to "only" chemistry, from the common cold and all mental disease to the religious instinct. Surely there are more levels of organization between human ethology and DNA than there are between DNA and quantum electrodynamics, and each level can require a whole new conceptual structure.

In closing, I offer two examples from economics of what I hope to have said. Marx said that quantitative differences become qualitative ones, but a dialogue in Paris in the 1920's sums it up even more clearly:

FITZGERALD: The rich are different from us.

HEMINGWAY: Yes, they have more money.

\section{References}

1. V. F. Weisskopf, in Brookhaven Nat. Lab. Publ. 8887360 (1965). Also see Nuovo Ci mento Suppl. Ser 1 4, 465 (1966); Phys. Today mento Suppl. Ser 1 4,
20 (No. 5), 23 (1967).

2. A. Bohr and B. R. Mottelson, Kgl. Dan Vidensk. Selsk. Mat. Fys. Medd. 27, 16 (1953) 3. Broken symmetry and phase transitions: I D. Landau, Phys. Z. Sowjetunion 11, 26, 542 (1937). Broken symmetry and collective motion zeneral: J. Goldstone, A. Salam, S. Weinberg, Phys, Rev. 127, 965 (1962); P. W. Anderson, Concepts in Solids (Benjamin, New York 1963), pp. 175-182; B. D. Josephson, thesis, Trinity College, Cambridge University (1962). Special cases: antiferromagnetism, P. W. Anderson, Phys, Rev, 86, 694 (1952); super conductivity, R. Rid. 110, 827 (1958) ibid. 112 1900 (1958); Y. Nambu, ibid. 117, 648 (1960)

4. F. London, Superfuids (Wiley, New York 1950), vol. 1

5. M. H. Cohen, J. Theor. Biol. 31, 101 (1971)

6. J. Clarke, Amer. J. Phys. 38, 1075 (1969); P. W. Anderson, Phys. Today 23 (No. 11), 23 (1970).

7. A. B. Pippard, Reconciling Physics with Reality (Cambridge Univ. Press, London, 1972). 